\title{
EVALUACIÓN DEL NIVEL DE RUIDO EMITIDO POR EL EQUIPO MECÁNICO UTILIZADO EN LA CONSTRUCCIÓN DE VÍAS DE CONCRETO EN DESAGUADERO, PERÚ 2020
}

\author{
EVALUATION OF THE NOISE LEVEL EMITTED BY THE MECHANICAL EQUIPMENT USED IN THE \\ CONSTRUCTION OF CONCRETE ROADS IN DESAGUADERO, PERU 2020
}

\author{
Samuel Huaquisto Cáceres ${ }^{1}$ \\ https://orcid.org/0000-0002-9294-6359 \\ shuaquisto@unap.edu.pe \\ Isabel Griscelda Chambilla Flores ${ }^{2}$ \\ (iD) https://orcid.org/0000-0002-1005-4964 \\ isa 1121@hotmail.com
}

Aceptado: 07/05/2021

Publicado online:30/05/2021

\begin{abstract}
RESUMEN
La contaminación sonora durante el proceso constructivo de un pavimento rígido es un factor ambiental que incide negativamente en la calidad de vida de los trabajadores y la población aledaña, en tal sentido, el objetivo del estudio fue analizar los niveles de ruido emitidos por el equipo mecánico utilizado en la construcción de vías urbanas a fin de proponer protección auditiva a los trabajadores. Los resultados indican que el equipo mecánico utilizado en la construcción de vías del distrito de Desaguadero emite niveles de ruido desde $69,6 \pm 5,1 \mathrm{dBA}$ a 98,4 $43,8 \mathrm{dBA}$, siendo los equipos livianos los más influyentes. La tarea más ruidosa es la del corte del concreto en pavimento que presenta un nivel de ruido de $87,88 \pm 3,2 \mathrm{dBA}$ medido a un radio de cinco metros de la fuente. En conclusión, el equipo mecánico utilizado en la construcción de vías emite niveles de ruido que pueden incidir en la salud de la población y en el desempeño de los trabajadores, recomendándose el uso de protectores auditivos de un nivel mínimo NRR $15 \mathrm{~dB}$ para operadores de equipo mecánico pesado y NRR $25 \mathrm{~dB}$ para equipos livianos.
\end{abstract}

\footnotetext{
1 Universidad Nacional del Altiplano Puno. Escuela profesional de Ingeniería. Dr. en Ciencia Tecnología y Medio Ambiente Puno, Perú.

2 Dirección Regional de Educación Puno. Unidad de gestión Educativa Local. Puno Perú. Msc. en Educación con mención en Administración de la Educación, Universidad Nacional del Altiplano Puno.
} 
Palabras clave: decibel; equipo mecánico; presión sonora; ruido; sonómetro; pavimento rígido.

\section{ABSTRACT}

Noise pollution during the construction process of a rigid pavement is an environmental factor that negatively affects the quality of life of workers and the surrounding population, in this sense, the objective of the study was to analyze the noise levels emitted by mechanical equipment. used in the construction of urban roads in order to offer hearing protection to workers. The results indicate that the mechanical equipment used in the construction of roads in the Desaguadero district emits noise levels from $69.6 \pm 5.1 \mathrm{dBA}$ to $98.4 \pm 3.8 \mathrm{dBA}$, with light equipment being the most influential. The loudest task is cutting concrete in pavement which exhibits a noise level of $87.88 \pm 3.2$ $\mathrm{dBA}$ measured at a radius of five meters from the source. In conclusion, the mechanical equipment used in the construction of roads emits noise levels that can affect the health of the population and the performance of workers, recommending the use of hearing protectors with a minimum level NRR $15 \mathrm{~dB}$ for equipment operators heavy mechanical and $25 \mathrm{~dB}$ NRR for light equipment.

Keywords: decibel; mechanical equipment; sound pressure; noise; sound level meter; rigid pavement.

\section{INTRODUCCIÓN}

Hace unos 2500 años ya había informes de sordera sobre personas que vivían cerca de las cataratas del río Nilo debido a que permanecían cerca de la fuente de ruido producido por la cascada. Durante la Revolución Industrial (siglo XVIII) el ruido se ha convertido en un subproducto del progreso a través de la difusión de grandes máquinas y vehículos en la producción de bienes y servicios (D'Agosto, 2019). Por tanto, el ruido a lo largo de la historia de la humanidad ha influido en la sociedad y ha causado problemas de salud a la población. Hoy en día, el ruido es uno de los principales contaminantes acústicos de la sociedad moderna, el cual incide directamente sobre el bienestar del entorno donde se genera; siendo principalmente causado por el tráfico vehicular, las actividades de construcción y recreativas (Salas López \& Barbosa Castillo, 2016), además de las actividades industriales, comerciales y el tráfico aéreo. En el caso de las actividades de construcción de vías urbanas, el ruido emitido por los equipos mecánicos de construcción es el contaminante más barato de producir y necesita muy poca energía para ser emitido (González Sánchez \& Fernández Díaz, 2014), no deja rastro como otros contaminantes y afecta negativamente a la calidad de vida de la población que conviven en ese entorno.

Un aspecto fundamental del desarrollo urbanístico de las ciudades es mejorar su infraestructura del transporte, lo que implica la construcción de vías en las que necesariamente se opera con equipo mecánico los cuales emiten niveles de ruido superiores a los especificados por la normativa y afectan directamente a la tranquilidad y salud auditiva de las personas que están propensos a sufrir factores de riesgo como la presión arterial y el estrés, influyendo en la salud física y mental de la población quienes tienen que lidiar con el ambiente ruidoso durante el proceso operativo de la maquinaria de construcción. Por otro lado, los trabajadores del sector construcción que operan la maquinaria están sujetos a riesgos laborales de pérdida de la audición debido a la asignación y uso inadecuado del equipo de protección auditiva no acorde al nivel de exposición el cual en muchos casos no cumple con las especificaciones dadas por los organismos competentes, lo que puede incidir en la disminución del desempeño laboral.

En ese sentido, el estudio tiene el propósito de determinar los niveles de ruido emitido por el equipo mecánico con el fin de analizar las incidencias en los trabajadores y la población en general. Para 
el caso de los trabajadores se les debe brindar acciones pertinentes de mitigación haciendo uso de protectores auditivos adecuados al entorno ruidoso y para la población del entorno, la aplicación y puesta en marcha de barreras acústicas coherentes con las horas pico donde el ruido supera los niveles máximos permisibles.

\section{La contaminación acústica}

La contaminación acústica es cualquier cambio en las propiedades físicas del medio ambiente causado por los sonidos de conjugación, deseables o no, que son directa o indirectamente perjudiciales para la salud, la seguridad y el bienestar de los seres vivos. Es necesario diferenciar sonido de ruido. El sonido es una perturbación que se propaga a través de un medio elástico (aire, agua, etc.) a una velocidad característica de ese medio, sin embargo, el ruido puede definirse como un sonido desagradable, un sonido que molesta a las personas (D’Agosto, 2019). La catalogación de un sonido concreto como ruido recae, en cierto modo, en la subjetividad individual del sujeto perceptor, el mismo sonido puede significar algo agradable, indiferente o insoportable, incluso para el mismo individuo (Martínez Suárez \& Moreno Jiménez, 2005).

Así como el ruido es definido como un sonido no agradable que origina sensaciones molestas e interfiere con la actividad humana (Ballesteros et al., 2012), estas son las formas de energía potencialmente nocivas en el ambiente (Amable Álvarez et al., 2017). En el caso del sonido, el contaminante no es una sustancia química sino el fenómeno físico caracterizado por la amplitud, frecuencia y patrón temporal (Cattaneo et al., 2013). Esta contaminación, afecta el ambiente y el paisaje sonoro de ciudades, paradójicamente de forma discreta, pero con importantes repercusiones en la salud y calidad de vida de las personas (Cohen \& Castillo, 2017).

\section{Influencia del ruido en la población}

Son conocidas las consecuencias que acarrea para la salud física y mental de la población exponerse al ruido, los cuales pueden ser: Efectos auditivos, como interferencia en la comunicación oral, en casos extremos, sordera; efectos no auditivos, como aumento de la presión arterial, taquicardia, jaquecas. Durante la exposición prolongada a más de $85 \mathrm{~dB}$ se han observado síntomas como gastritis, colitis, aumento de la glucemia y la colesterolemia, en niños influye en los efectos de atención, retardo del aprendizaje, aislamiento y poca sociabilidad (Cattaneo et al., 2013), además, puede: alterar el sueño, aumentar niveles de estrés, disminuir el rendimiento del trabajador, provocar inestabilidad emocional y fatiga. En el caso de mujeres expuestas a la contaminación ambiental acústica tienen un mayor riesgo de preeclampsia (Auger et al., 2018).

En el trabajo, la exposición al ruido ocasiona disminuciones de sensibilidad auditiva. En algunas pruebas de habilidad, se ha demostrado que, con la exposición al ruido continuo, existe disminución del rendimiento y eficiencia, elevando el número de errores, y un probable aumento de accidentes como consecuencia de la reducción de la habilidad (Degrandi \& Nogueira, 2012). Así, la exposición a los riesgos ocupacionales puede ocasionar pérdida en la calidad y desempeño del trabajador, y determina su comportamiento, su calidad de vida y salud, incluyendo los acontecimientos por enfermedad y accidentes de trabajo (Ganime et al., 2010).

\section{Niveles de ruido según estándares peruanas e internacionales}

En el Perú, la legislación vigente establece Estándares Nacionales de Calidad Ambiental refrendado por el Decreto DS 024-2016-EM en el que se establece los niveles de ruido de acuerdo al tiempo de exposición, el cual se muestra en la tabla 1; además, se tiene el DS N085-2003-PCM que establece los niveles de ruido según zonas y horarios, así como de protección, residencial, comercial o industrial presentado en la tabla 2. 
Tabla 1

Niveles de Ruido Establecidos por el MINEM.

\begin{tabular}{lc}
\hline $\begin{array}{l}\text { Escala de } \\
\text { Ponderación "A" }\end{array}$ & $\begin{array}{c}\text { Tiempo de Exposición Máximo } \\
\text { en Una Jornada Laboral }\end{array}$ \\
\hline 82 decibeles & 16 horas/día \\
83 decibeles & 12 horas/día \\
85 decibeles & 8 horas/día \\
88 decibeles & 4 horas/día \\
91 decibeles & $11 / 2$ horas/día \\
94 decibeles & 1 hora/día \\
97 decibeles & $1 / 2$ hora/día \\
100 decibeles & $1 / 4$ hora / día \\
\hline Nota: Anexo 12 del DS 024-2016-MINEM (MINEM, 2016).
\end{tabular}

Tabla 2

Estándares de Calidad Ambiental para Ruido Determinados por la PCM.

\begin{tabular}{|c|c|c|}
\hline \multirow{3}{*}{ Zonas de Aplicación } & \multicolumn{2}{|c|}{ Valores de $\mathrm{L}_{\text {AeqT }}$} \\
\hline & Horario & Horario \\
\hline & Diurno & Nocturno \\
\hline Zona de & 50 & 40 \\
\hline \multicolumn{3}{|l|}{ Especial } \\
\hline Zona Residencial & 60 & 50 \\
\hline Zona Comercial & 70 & 60 \\
\hline Zona Industrial & 80 & 70 \\
\hline
\end{tabular}

En temática internacional sobre niveles de ruido resaltan instituciones como la Agencia de Protección del Medio Ambiente (EPA), el Consejo Internacional de Ruido (INC), el Instituto Nacional para la Seguridad y Salud Ocupacional (NIOSH), Administración de Seguridad y Salud Ocupacional (OSHA) y la Organización Mundial de la Salud (OMS) (Garrido Galindo et al., 2015). Además, las especificaciones sobre niveles de ruido dadas por la OMS se resumen en la tabla 3 para la región europea, en la que se establecen parámetros máximos para los tipos de ruido según su exposición media o nocturna, los cuales deben ser referentes para nuestra región.

Tabla 3

Valores Referenciales de Ruido Según la OMS para la Región de Europa.

\begin{tabular}{|c|c|c|}
\hline Tipos & Exposición Media al Ruido & Exposición Nocturna al Ruido \\
\hline $\begin{array}{l}\text { Ruido del } \\
\text { tráfico } \\
\text { rodado }\end{array}$ & $\begin{array}{l}\text { Se recomienda reducir niveles de ruido por } \\
\text { debajo de } 53 \text { decibeles }(\mathrm{dB}) \mathrm{L}_{\text {den, }} \text { el ruido } \\
\text { del tráfico por encima de este nivel está } \\
\text { asociado con efectos adversos para la } \\
\text { salud. }\end{array}$ & $\begin{array}{l}\text { Se recomienda reducir el ruido por debajo de } \\
45 \mathrm{~dB} \mathrm{~L}_{\text {noche, }} \text { el ruido del tráfico por la noche } \\
\text { por encima de este nivel se asocia con efectos } \\
\text { adversos sobre el sueño. }\end{array}$ \\
\hline $\begin{array}{l}\text { Ruido } \\
\text { ferroviario }\end{array}$ & $\begin{array}{l}\text { Se recomienda reducir niveles de ruido por } \\
\text { debajo de } 54 \mathrm{~dB} \mathrm{~L}_{\text {den, }} \text { como ruido } \\
\text { ferroviario por encima de este nivel se } \\
\text { asocia con efectos adversos para la salud. }\end{array}$ & $\begin{array}{l}\text { Se recomienda reducir el ruido por debajo de } \\
44 \mathrm{~dB} \text { Lnoche, el ruido ferroviario nocturno por } \\
\text { encima de este nivel está asociado con efectos } \\
\text { adversos en el sueño. }\end{array}$ \\
\hline $\begin{array}{l}\text { Ruido de } \\
\text { aeronaves }\end{array}$ & $\begin{array}{l}\text { Se recomienda reducir el ruido por debajo } \\
\text { de } 45 \mathrm{~dB} L_{\text {den, ya que el ruido de los aviones }} \\
\text { por encima de este nivel está asociado con } \\
\text { efectos adversos para la salud. }\end{array}$ & $\begin{array}{l}\text { Se recomienda encarecidamente reducir el } \\
\text { ruido por debajo de } 40 \mathrm{~dB} \text { Lnoche, el ruido de las } \\
\text { aeronaves por encima de este nivel se asocia } \\
\text { con efectos adversos en el dormir. }\end{array}$ \\
\hline $\begin{array}{l}\text { Ruido de } \\
\text { turbinas } \\
\text { eólicas }\end{array}$ & $\begin{array}{l}\text { Se recomienda condicionalmente reducir } \\
\text { los niveles de ruido por debajo de } 45 \mathrm{~dB} \\
\text { Lden, ya que el ruido de las turbinas eólicas } \\
\text { por encima de este nivel está asociado con } \\
\text { efectos adversos para la salud. }\end{array}$ & $\begin{array}{l}\text { No se hace ninguna recomendación para la } \\
\text { exposición nocturna promedio al ruido } L_{\text {noche }} \\
\text { de turbinas eólicas. El ruido de la turbina es } \\
\text { demasiado bajo para permitir una } \\
\text { recomendación. }\end{array}$ \\
\hline $\begin{array}{l}\text { Ruido de } \\
\text { ocio }\end{array}$ & $\begin{array}{l}\text { Se recomienda condicionalmente reducir el } \\
\text { promedio anual de todas las fuentes de } \\
\text { ruido de ocio combinadas a } 70 \mathrm{~dB} \mathrm{~L}_{\text {Aeq }}, 24 \mathrm{~h} \\
\text { ya que el ruido de ocio por encima de este } \\
\text { nivel se asocia con efectos adversos para la } \\
\text { salud. }\end{array}$ & $\begin{array}{l}\text { Para exposiciones al ruido de un solo evento y } \\
\text { de impulso, se recomienda seguir las pautas y } \\
\text { regulaciones legales existentes para limitar el } \\
\text { riesgo de aumento de la discapacidad auditiva } \\
\text { por ruido de ocio en niños y adultos. }\end{array}$ \\
\hline
\end{tabular}

Nota: Elaborado en base a lo recomendado por la OMS (WHO, 2018), donde $\mathrm{L}_{\text {den }}$ : nivel de ruido medio durante el día, tarde y noche; $y \mathrm{~L}_{\text {noche }}$ en el nivel de ruido durante la noche. 
Complementariamente a las especificaciones indicadas en los acápites anteriores, se precisa que, en cuanto a fábricas que usan máquinas de una o varias unidades causarían un sonido de aproximadamente $98 \mathrm{~dB}$ y más, los aviones causan el sonido más alto entre todos con $150 \mathrm{~dB}$, los vehículos de carretera también son grandes contribuyentes de la contaminación acústica, estos vehículos incluyen camiones, autobuses, tractores e incluso motocicletas y la mayoría de los automóviles (Muralikrishna et al., 2017) con niveles de ruido variables según su funcionamiento y antigüedad.

\section{Ruido generado por el equipo mecánico}

El ruido mecánico existe en todas las industrias a consecuencia del funcionamiento de las máquinas, algunas de ellas dotadas de menos tecnología producen ruidos excesivos más allá de lo tolerable (Ganime et al., 2010). En un estudio sobre análisis de ruido en empresas manufactureras en Nigeria se determinó que las máquinas generadoras de electricidad produjeron el mayor porcentaje total de ruido de aproximadamente $26,7 \%$, mientras que, para compresor de aire, caldera, máquina de prensado y otros fueron de 18,$9 ; 16,7 ; 16,7$ y $21,0 \%$, respectivamente (Bolaji et al., 2018), lo que implica que la maquinaria pesada genera la mayor cantidad de ruido en todo tipo de industrias.

En el caso de la industria de la construcción, un factor social y ambiental implícito comúnmente relacionado es la contaminación acústica resultante de las actividades que se realizan durante las diversas etapas de construcción (Hammad et al., 2016). En un estudio de ruido en construcciones modulares, el nivel de exposición al ruido de los trabajadores cuando operan tres estaciones de trabajo principales era alrededor de $86 \mathrm{dBA}$, siendo los trabajadores en la estación de revestimiento los que están expuestos al mayor nivel de ruido (Dabirian et al., 2020). En otro estudio se desarrolló una base de datos de niveles de ruido de equipos que se usan en la construcción, los niveles sonoros en $\mathrm{dB}(\mathrm{A})$ emitidos por distintos equipos a 2 m de distancia son: sonda vibratoria $5 \mathrm{Hp} \mathrm{91,8;} \mathrm{trompo} \mathrm{mezclador} \mathrm{de} \mathrm{5,5} \mathrm{Hp} \mathrm{89,9;} \mathrm{sierra}$ circular 1400 w 99,6; placa compactadora $5 \mathrm{Hp} \mathrm{99,8;} \mathrm{rodillo} \mathrm{compactador} 8 \mathrm{Hp} \mathrm{89,5;} \mathrm{motobomba} \mathrm{7,5} \mathrm{Hp}$ 88; martillo neumático y compresor 8 bar 105,5 y generador trifásico 12 Hp 86,6 (Mosquera, 2003). Así mismo, en estudios realizados sobre la aplicación del Método William Fine para la evaluación de riesgos laborales en motoniveladoras, cargadoras y bulldozers, se ha determinado niveles de ruido en las etapas de máquina encendida, en movimiento, acelerado moderado y acelerado máximo con valores máximos y dosis de 7 horas de trabajo de: la motoniveladora 81,0 dB(A) con dosis 0,48; el cargador con 82,7 dB(A) a una dosis de 0,57 y el bulldozers de 96,9 dB(A) con dosis 3,63 (Rodrigo Fernando, 2013).

\section{METOdOLOGÍA}

El lugar de estudio se ubica en la zoma comercial del distrito de Desaguadero de la provincia de Chucuito en el departamento de Puno-Perú a una altitud media de $3830 \mathrm{msnm}$ adyacente al Lago Titicaca en la frontera Perú-Bolivia, el cual se muestra en la figura 1. En este ámbito se han construido cinco vías donde se ha utilizado equipo mecánico pesado y liviano para su pavimentación con concreto en el año 2019, dichas vías son: Cusco, en el intervalo de progresivas 0+000 - 0+733.10; Leticia, progresiva 0+185 0+652.00; Pachacutec, progresiva 0+000 - 0+169.00; Gerardo Pérez, progresiva 0+000 - 0+249.30 y José Antonio Encinas, progresiva 0+000-0+190.30.

Para realizar el monitoreo del nivel de ruido se utilizó un Sonómetro tipo 2 PRASEK modelo PR 352 para el tiempo de funcionamiento del equipo mecánico, cuyas diferencias han sido como máximo 3 dB de acuerdo a la NTP-ISO 9612 2010. En la figura 2 se observa proceso de monitoreo realizado a los trabajos en el pavimento de concreto.

Los equipos mecánicos monitoreados con una antigüedad máxima de 5 años, son los mostrados en las tablas 4 y 5 , cuyos resultados de niveles de ruido interesan para determinar el nivel de protección 
auditiva del operador y la tabla 6 muestra los resultados de niveles de ruido de los trabajos realizados en que se utiliza una combinación de equipos mecánicos.

Figura 1

Localización de la zona de estudio. Desaguadero, PunoPerú. Vista extraída de Google Maps

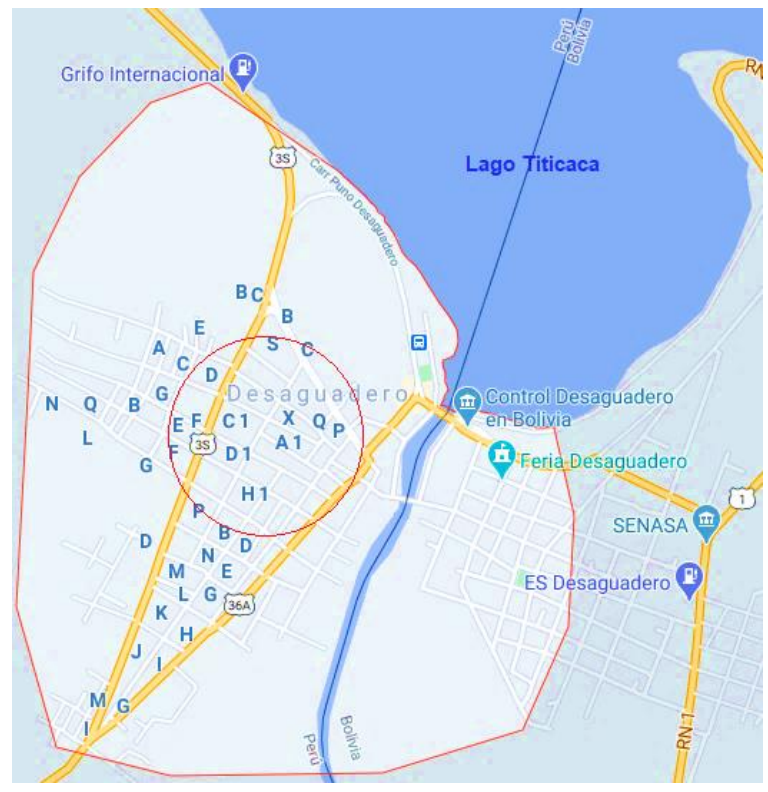

\section{Figura 2}

Monitoreo realizado a la cortadora de concreto en operación con sonómetro tipo 2.

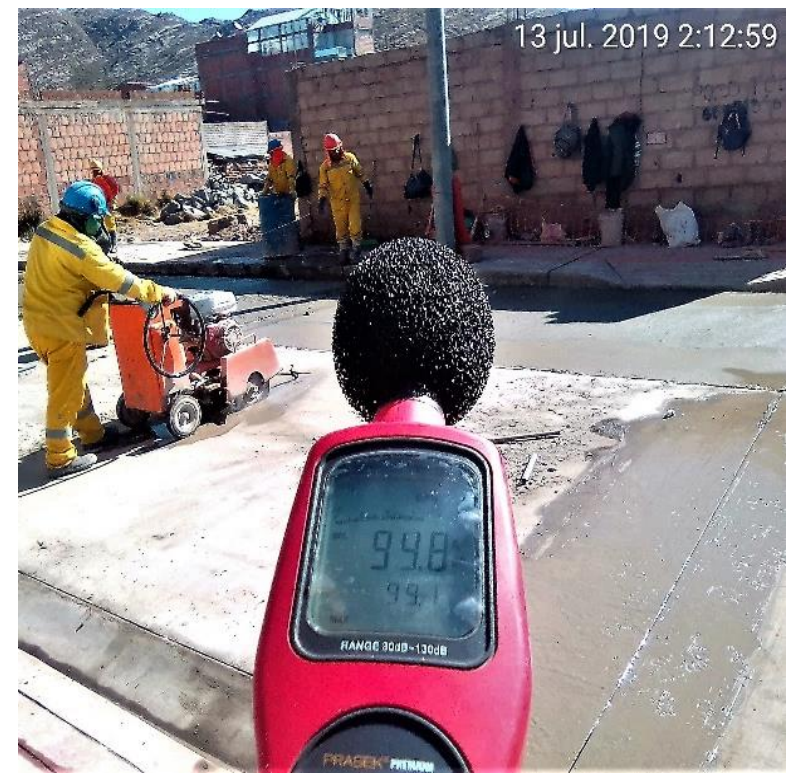

Los cálculos han sido realizados de acuerdo a la Norma Técnica Peruana (NTP, 2010) del que se ha considerado la ecuación para determinar el nivel de ruido ponderado " $A$ " ( $\left.L_{p, A, \text { eqT }}\right)$ dada por la ecuación (1), el cual está en función al nivel de ruido continuo equivalente ponderado " $A$ " durante una tarea de duración " $T_{m}$ " ( $\left.L_{p, A, e q T, m i}\right)$, al número de una medición de la tarea " $m$ " (i) y al número total de mediciones de la tarea (I).

$$
L_{p, A, e q T}=10 \log \left(\frac{1}{I} \sum_{i=1}^{I} \quad 10^{0.1 x L_{p, A, e q T, m i}}\right) d B(1)
$$

El nivel de exposición al ruido ponderado " $A$ " normalizado para una jornada laboral diaria de 8 horas $\left(L_{E x, 8 h}\right)$ se determina con la ecuación (2), que depende del nivel de ruido continuo equivalente ponderado " $A$ " ( $\left.L_{p, A, e q T e}\right)$, de la duración efectiva en horas de la jornada laboral $\left(T_{e}\right)$ y de la duración total de la jornada laboral ( $\left.T_{0}=8 \mathrm{~h}\right)$.

$$
L_{E X, 8 h}=L_{p, A, e q T e}+10 \lg \left[\frac{T_{e}}{T_{o}}\right] d B
$$

La incertidumbre estándar basada en la función se determina a partir de la ecuación (3) en la que $\mathrm{u}_{1}$ es la incertidumbre estándar debida al muestreo del nivel de ruido igual a la desviación estándar de la muestra determinado con la ecuación (4), $\mathrm{u}_{2}$ es la incertidumbre debido a los instrumentos utilizados igual a $1.5 \mathrm{~dB}$ para el sonómetro de clase $2, \mathrm{u}_{3}$ es la incertidumbre debido a la posición del micrófono igual a 1 $\mathrm{dB}$, los coeficientes de sensibilidad $\mathrm{c}_{1}$ y $\mathrm{c}_{2}$ son determinados a partir de la NPT con $\mathrm{c}_{2}$ igual a 1. 


$$
\begin{gathered}
U=1.65 \sqrt{c_{1}^{2} u_{1}^{2}+c_{2}^{2}\left(u_{2}^{2}+u_{3}^{2}\right)} \\
u_{1}=\sqrt{\frac{1}{N-1}\left[\sum_{n=1}^{N}\left(L_{p, A, e q T, n}-\underline{L}_{p, A, e q T}\right)^{2}\right]}
\end{gathered}
$$

Además, la dosis de ruido se determina para el tiempo de 8 horas y nivel de ruido permitido de $85 \mathrm{dBA}$, como lo muestra la ecuación (5), en función al tiempo de exposición del trabajador (T) y al nivel equivalente de ruido en la escala de ponderación " $A$ " (L).

$$
\% \text { dosis }=\left(\frac{T}{8}\right) 2^{(L-85) / 3}
$$

Complementando a las ecuaciones anteriores, el nivel de ruido atenuado (NRA) se calcula según el ponderado "A" de la OSHA determinado por la ecuación (6), en función al nivel de presión sonoro (NPS) que es el nivel de ruido contemplado por la ecuación (1) y al nivel de reduccion del ruido (NRR). En el estudio se presentan las recomendaciones correspondientes para un protector auditivo del tipo copa, que es el más usado en la construcción.

$$
N R A=N P S-0.75(N R R-7 d B A)
$$

\section{RESULTADOS}

\section{Niveles de ruido y dosimetrías medidos en el equipo mecánico}

Los niveles de ruido para cada equipo mecánico individual han sido medidos a una distancia promedio de $2 \mathrm{~m}$ y una altura de 1,5 $\mathrm{m}$ de la fuente de emisión, controlando las interferencias por otros agentes en el tiempo de trabajo del equipo monitoreado durante su funcionamiento. Los resultados que involucran al operador del equipo han sido agrupados en la tabla 4 para equipo mecánico pesado y en la tabla 5 para equipo mecánico liviano, las mismas que contemplan los NRR para el uso de protectores auditivos para el operador del equipo mecánico, considerando un nivel de ruido atenuado (NRA) de 85 db para una jornada de trabajo de 8 horas.

\begin{tabular}{|c|c|c|c|c|c|}
\hline Equipo Mecánico Pesado & $\begin{array}{l}L_{p, A, e q T} \\
(d B A)\end{array}$ & $\begin{array}{c}\text { Incertidumbre } \\
\mathrm{U}(\mathrm{dBA})\end{array}$ & $\begin{array}{l}\text { Tiempo } \\
\text { (horas) }\end{array}$ & $\begin{array}{c}\text { Dosis } \\
\%\end{array}$ & $\begin{array}{r}\text { NRR } \\
(\mathrm{dBA})\end{array}$ \\
\hline Rodillo SINOMACH CMD512D & 85,4 & 3,8 & 3 & 41 & 8 \\
\hline Retroexcavadora CAT 420 F2 & 77,8 & 5,9 & 6 & 14 & - \\
\hline Autohormigonera $3.5 \mathrm{~m}^{3} \mathrm{DIECI} 14700$ & 84,9 & 4,3 & 6 & 74 & 7 \\
\hline Minicargador BODCAT S630 & 87,5 & 4,7 & 7 & 156 & 11 \\
\hline Motoniveladora KOMATSU GD 405 A-3 & 82,7 & 4,3 & 4 & 30 & 4 \\
\hline Excavadora CAT 330BL & 80,5 & 4,0 & 7 & 31 & - \\
\hline Camión Mixer 8m³ HOWO A7 & 90,5 & 3,6 & 6 & 265 & 15 \\
\hline Camión Cisterna VOLVO N 33 & 69,6 & 5,1 & 4 & 1 & - \\
\hline Volquete VOLVO NL12 & 79,8 & 3,8 & 4 & 15 & - \\
\hline
\end{tabular}

\section{Tabla 4}

Niveles de Ruido y Dosimetrías para el Equipo Mecánico Pesado Común Utilizado en la Construcción del Pavimento Rígido de Concreto, Distrito de Desaguadero.

Nota: $L_{p, A, \text { eqT }}$ es determinado con la ecuación (1), U con la ecuación (3), el \% de dosis con la ecuación (5) y NRR determinado con la ecuación (6). 
Los niveles de ruido, incertidumbre y dosis de trabajo para cada equipo mecánico pesado son mostrados en la tabla 4, en la que los niveles de ruido analizados varían desde un valor mínimo de 69,6 $\pm 5,1$ dBA para el camión cisterna hasta un valor máximo de 90,5 $\pm 3,6$ dBA para el camión mixer. Las dosis de trabajo para el tiempo medido a los cuales están expuestos los operadores de cada equipo pesado, varían desde $1 \%$ hasta $265 \%$; tanto el minicargador y el camión mixer superan el $100 \%$ de dosis de exposición al operario; por tanto, se recomienda el uso de protectores auditivos tipo copa de un NRR bajo o medio, mínimo de 15 dBA OSHA.

\section{Tabla 5}

Niveles de Ruido y Dosimetrías para el Equipo Mecánico Liviano Utilizado en la Construcción del Pavimento Rígido de Concreto, Distrito de Desaguadero.

\begin{tabular}{|c|c|c|c|c|c|}
\hline Equipo Mecánico Liviano & $\begin{array}{l}L_{p, A, e q T} \\
(d B A)\end{array}$ & $\begin{array}{c}\text { Incertidumbre } \\
\mathrm{U}(\mathrm{dBA})\end{array}$ & $\begin{array}{l}\text { Tiempo } \\
\text { (horas) }\end{array}$ & $\begin{array}{c}\text { Dosis } \\
\%\end{array}$ & $\mathrm{NRR}(\mathrm{dBA})$ \\
\hline Rotomartillo & 92,1 & 5,1 & 2 & 129 & 17 \\
\hline Motobomba & 90,1 & 5,1 & 3 & 123 & 14 \\
\hline Compresora & 79,2 & 3,1 & 7 & 23 & - \\
\hline Amoladora & 98,4 & 3,8 & 2 & 548 & 25 \\
\hline Generador trifásico & 90,8 & 4,3 & 6 & 286 & 15 \\
\hline Cortadora de concreto TH420 & 96,2 & 5,5 & 7 & 1174 & 22 \\
\hline Vibradora de concreto & 89,2 & 4,3 & 4 & 131 & 13 \\
\hline Plancha compactadora & 95,5 & 3,8 & 3 & 427 & 21 \\
\hline
\end{tabular}

En la tabla 5, se presenta los niveles de ruido ponderado A para cada equipo mecánico liviano común que se utiliza en la construcción de un pavimento rígido y las correspondientes incertidumbres y dosis de trabajo. Los niveles ruido monitoreados varían desde 79,2 $\pm 3,1 \mathrm{dBA}$ para la compresora hasta un valor máximo de $98,4 \pm 3,8$ dBA para la amoladora. Las dosis de trabajo a los cuales están expuestos los operadores de cada equipo mecánico liviano varían desde $23 \%$ hasta $1174 \%$; solo un equipo mecánico liviano no excede la dosis de exposición a más del 100 \% que corresponde a la compresora, todos los demás equipos como el rotomartillo, motobomba, amoladora, generador trifásico, cortadora de concreto, vibradora de concreto y plancha compactadora exceden la dosis del $100 \%$ de exposición del operario; en consecuencia, se recomienda el uso de un protector auditivo tipo copal de un NRR medio a alto, mínimo de 25 dBA OSHA.

\section{Niveles de ruido y dosimetrías por tareas específicas.}

El nivel de ruido medido para cada tarea principal que conforma la estructura de un pavimento rígido con uso de equipo mecánico específico en un radio promedio de $5 \mathrm{~m}$ de la fuente sonora dentro del cual un trabajador realiza labores se muestra en la tabla 6. La maquinaria principal utilizada en las diferentes etapas son: en corte a nivel de subrasante, excavadora 330B y volquetes NL12; en conformación de subrasante y subbase granular, el volquete NL12, la motoniveladora GD 405 A-3, el camión cisterna N33 y rodillo vibratorio CMD 512D; en conformación de base granular para veredas, minicargador S630, camión cisterna N33 y plancha compactadora; en concreto para veredas autohormigonera de $3,5 \mathrm{~m}^{3}$, camión mixer $8 \mathrm{~m}^{3}$ y vibrador de concreto; en concreto para pavimento rígido, minicargador 5630 , autohormigonera de $3,5 \mathrm{~m}^{3}$, camión mixer $8 \mathrm{~m}^{3}$ y vibrador de concreto; en corte para pavimento se tiene a la cortadora de concreto. La etapa de la elaboración del concreto premezclado en planta implica la utilización de un camión mixer $8 \mathrm{~m}^{3}$, la retroexcavadora $420 \mathrm{~F} 2$ y la motobomba; finalmente, existe una 
etapa complementaria que es el pintado en pavimento el cual utiliza una compresora y un generador trifásico.

Analizando la tabla 6, las tareas de la construcción de un pavimento están limitadas a una jornada laboral de 8 horas. Se observa que los niveles de ruido equivalente $A$ promedio varían desde 71,99 $\pm 3,0$ $\mathrm{dBA}$, para la tarea de pintado en pavimento, hasta $87,88 \pm 3,2 \mathrm{dBA}$ para la tarea de corte de concreto en pavimento; así mismo el porcentaje de dosis a los cuales están expuestos los trabajadores presentan valores que van desde $5 \%$ para pintado en pavimento hasta $194 \%$ para corte de concreto en pavimento excediéndose la dosis del $100 \%$.

\section{Tabla 6}

Niveles de Ruido por Tareas y Partidas Específicas Realizadas con Equipo Mecánico en la Construcción del Pavimento rígido.

\begin{tabular}{|c|c|c|c|c|c|c|}
\hline Tareas & Partidas & $\begin{array}{c}T_{\text {prom }} \\
\text { (horas) }\end{array}$ & $\begin{array}{l}L_{p, A, \text { eq }} \\
\text { (dBA) }\end{array}$ & $\begin{array}{l}\text { Lex,8h } \\
\text { (dBA) }\end{array}$ & $\begin{array}{c}\text { Incertidumbre } \\
\mathrm{U}(\mathrm{dBA})\end{array}$ & $\begin{array}{c}\text { Dosis } 8 \mathrm{~h} \\
\%\end{array}$ \\
\hline \multirow{2}{*}{$\begin{array}{l}\text { Corte a nivel de } \\
\text { subrasante }\end{array}$} & Planificación & 1 & 65,66 & \multirow[b]{2}{*}{75,20} & \multirow[b]{2}{*}{3,2} & \multirow[b]{2}{*}{10} \\
\hline & $\begin{array}{l}\text { Excavación y } \\
\text { eliminación }\end{array}$ & 7 & 75,72 & & & \\
\hline \multirow{3}{*}{$\begin{array}{l}\text { Conformación } \\
\text { subrasante y sub base } \\
\text { granular }\end{array}$} & $\begin{array}{l}\text { Traslado de } \\
\text { material }\end{array}$ & 2 & 71,60 & \multirow{3}{*}{79,86} & \multirow{3}{*}{3,0} & \multirow{3}{*}{30} \\
\hline & Conformación & 4 & 80,55 & & & \\
\hline & Compactación & 2 & 81,64 & & & \\
\hline \multirow{3}{*}{$\begin{array}{l}\text { Conformación } \\
\text { compactación de base } \\
\text { granular en veredas }\end{array}$} & Limpieza & 2 & 82,70 & \multirow{3}{*}{83,76} & \multirow{3}{*}{2,4} & \multirow{3}{*}{75} \\
\hline & Conformación & 3 & 83,03 & & & \\
\hline & Compactación & 3 & 84,90 & & & \\
\hline \multirow[t]{2}{*}{ Concreto en veredas } & $\begin{array}{l}\text { Vaciado de } \\
\text { concreto }\end{array}$ & 5 & 82,61 & \multirow[t]{2}{*}{80,62} & \multirow[t]{2}{*}{3,4} & \multirow[t]{2}{*}{36} \\
\hline & Acabado y pulido & 3 & 65,77 & & & \\
\hline \multirow[t]{3}{*}{ Concreto en pavimento } & Limpieza & 1 & 83,28 & \multirow{3}{*}{83,98} & \multirow{3}{*}{2,9} & \multirow{3}{*}{79} \\
\hline & Vaciado & 6 & 84,73 & & & \\
\hline & Acabado & 1 & 66,24 & & & \\
\hline \multirow{2}{*}{$\begin{array}{l}\text { Corte de concreto en } \\
\text { pavimento }\end{array}$} & Trazo & 2 & 65,67 & \multirow{2}{*}{87,88} & \multirow{2}{*}{3,2} & \multirow{2}{*}{194} \\
\hline & Corte de concreto & 6 & 89,12 & & & \\
\hline \multirow[t]{2}{*}{$\begin{array}{l}\text { Elaboración de mezclas } \\
\text { de concreto en planta }\end{array}$} & $\begin{array}{l}\text { Elaboración de } \\
\text { mezcla }\end{array}$ & 6 & 83,00 & \multirow[t]{2}{*}{81,77} & \multirow[t]{2}{*}{3,2} & \multirow[t]{2}{*}{47} \\
\hline & Limpieza & 2 & 65,15 & & & \\
\hline \multirow[t]{2}{*}{ Pintado en pavimento } & Trazo & 2 & 64,34 & \multirow{2}{*}{71,99} & \multirow{2}{*}{3,0} & \multirow{2}{*}{5} \\
\hline & Pintado & 6 & 73,05 & & & \\
\hline
\end{tabular}

Nota: $T_{\text {prom }}$ es el tiempo promedio de trabajo, $L_{p, A, \text { eqT }}$ es determinado con la ecuación (1), $L_{E x, 8 h}$ con la ecuación (2), U con la ecuación (3) y el \% de dosis con la ecuación (5).

\section{DISCUSIÓN}

Con respecto a los resultados de la tabla 4, se afirma que el entorno ambiental en el que se encuentran los operadores de equipos mecánicos de construcción es bastante ruidoso y potencialmente dañino para la salud si no se usa los protectores auditivos de forma adecuada; el rodillo, la autohormigonera, el minicargador y el camión mixer igualan o superan el límite superior de 85 dBA (Garrido Galindo et al., 2015), en el caso de los equipos mecánicos livianos los analizados en este estudio exceden este valor, 
excepto la compresora tal como se observa en la tabla 5 concordante con otros estudios realizados en equipos que se usan en la construcción en la que se superan el valor de 85 dBA (Mosquera, 2003), por tanto, el operador necesariamente debe usar equipos de protección personal adecuados, dentro de ellos los protectores auditivos tipo copa para reducir la exposición al ruido por el uso de equipos de construcción (Saleh et al., 2017). Situación similar se presenta en el sector minero que se asemeja al de la construcción, en un estudio realizado en Madrid se hallaron resultados elevados donde un $90,2 \%$ de los puestos de trabajo presentan un $\mathrm{L}_{\mathrm{Ex}, 8 \mathrm{~h}}$ superior a $80 \mathrm{dBA}$, un $75,4 \%$ superan los $85 \mathrm{dBA}$, y un $47,5 \%$ supera los 90 dBA de nivel diario de exposición sonora (Pavón García, 2007).

En cuanto a la incidencia del ruido generado por el uso de equipo mecánico en la población, como la zona de desaguadero es netamente comercial el límite superior es los $70 \mathrm{dBA}$ en horario diurno (WHO, 2018), los cuales son superados por todos los equipos livianos indicados en la tabla 5 y equipos pesados de la tabla 4, excepto por el camión cisterna el cual es prácticamente igualado en el nivel de presión sonora, por tanto, los efectos críticos en la salud de la población podrían ser el estrés, aumento de la presión arterial y la discapacidad auditiva en un tiempo prolongado de 24 horas para un entorno industrial, comercial y de tráfico (Berglund et al., 1999) ruidoso.

Así también, del análisis de las tablas 4 y 5 se deduce que gran parte de los trabajadores del proyecto están expuestos a una dosis que supera el $100 \%$ lo que concuerda con estudios realizados en trabajos de construcción en la que se ha determinado que la mayoría de ellos, 67,5 \%, sufren una exposición diaria que excede los $80 \mathrm{dBA}$; lo que es peor, es que 50,0\%, exceden los $87 \mathrm{dBA}$ (Fernández et al., 2009), por tanto, además de influir en la salud del trabajador puede ocasionar pérdida en la calidad y desempeño en el trabajo (Ganime et al., 2010) y los rendimientos pueden disminuir afectando los costos de la mano de obra.

En el caso de actividades de construcción en edificaciones, la etapa de excavación tiene niveles más altos y una mayor dependencia de la maquinaria utilizada, el cual se pueden asemejar a proyectos viales, en un estudio se han reportado resultados de emisión global de ruido a lo largo de todo el proceso constructivo con valores de 75,7 dB y 67,7 dBA con las descargas de concreto caracterizados por un mayor nivel de ruido (Ballesteros, et al., 2010) el cual difiere a los niveles de ruido de las tareas del presente estudio por ser puntual, en el caso del vaciado del concreto es de 84,73 dBA y para la elaboración del mismo 83,00 dBA como se indica en la tabla 6 . A nivel de conformación subrasante y sub base granular se obtuvo 79,86 dBA; resultados similares se han obtenido en estudios sobre trabajos de construcción en la etapa de excavación y movimiento de tierras, que proporcionan un nivel equivalente ponderado $A$ promedio de 81 dBA y dosis de ruido por debajo del $100 \%$, sugiriéndose diseñar una combinación adecuada de duración de las tareas a desarrollar por cada trabajador para no sobrepasar una dosis de ruido del $100 \%$ (Fernández et al., 2009).

En general, para la jornada laboral de 8 horas de trabajo se puede considerar aceptable la exposición al nivel de ruido en la mayoría de los casos, siempre y cuando el trabajador se mantenga a una distancia mayor a $5 \mathrm{~m}$ de la fuente y en el caso de las tareas muy ruidosas como la de corte del concreto se sugiere su mitigación son el uso adecuado de los protectores auditivos tipo copa el cual es regulado por la legislación laboral vigente. Por otro lado, estas tareas de construcción civil afectan a la población con niveles de ruido que superan los 70 dBA para zona comercial (WHO, 2018), lo que puede influir en efectos psicológicos como alteración del sueño, estrés, baja concentración y disminución del desempeño laboral y físico, asimismo, nerviosismo, fatiga e inestabilidad emocional (Cohen \& Castillo, 2017) debido al tiempo de ejecución que presentan los proyectos de infraestructura civil; por lo que se sugiere el uso de barreras acústicas, tal como se aplicó en un estudio sobre el diseño y construcción de la barrera acústica para reducir la contaminación auditiva generada por una perforadora de concreto en vías 
públicas (Espinoza Vasquez \& Jaramillo Arango, 2012), con el fin de no afectar a la salud de la población aledaña al proyecto en construcción.

\section{CONCLUSIONES}

El nivel de ruido que emite el equipo mecánico pesado utilizado en la construcción del pavimento rígido varía entre los valores de 69,6 $\pm 5,1 \mathrm{dBA}$ a 90,5 3,6 dBA, siendo las dosimetrías más críticas para el operador del minicargador y camión mixer, observándose además una prevalencia para el rodillo y autohormigonera por estar en el umbral de $85 \mathrm{dBA}$; por tanto, se sugiere la utilización adecuada de protectores auditivos tipo copa de un nivel mínimo de NRR 15 dBA para operadores de equipo mecánico pesado.

El equipo mecánico ligero reporta valores de niveles de ruido que superan los $85 \mathrm{dBA}$, exceptuando la compresora, llegando a niveles de $98,4 \pm 3,8$ dBA para la amoladora y $96,2 \pm 5,5$ dBA para la cortadora de concreto. Las dosimetrías presentan valores críticos que exceden el $100 \%$ para los operarios, en consecuencia, el uso de los protectores auditivos de forma adecuada para los operadores de equipo liviano es de vital importancia siendo recomendable los de tipo copa con un nivel mínimo de NRR 25 dBA. En las etapas de la construcción del pavimento rígido, las tareas reportan niveles de ruido que varían desde $71,99 \pm 3,0 \mathrm{dBA}$ hasta $87,88 \pm 32 \mathrm{dBA}$. La dosimetría crítica se presenta para la tarea de corte de concreto en pavimento que supera el $100 \%$ para una jornada de 8 horas de trabajo. Por tanto, estos niveles de presión sonora inciden en la salud mental de la población porque exceden los 70 dBA para zona comercial en horario diurno, sugiriéndose a los proyectistas incidir en la adopción de estrategias orientadas a disminuir el ruido mediante el uso de barreras acústicas.

\section{REFERENCIAS}

Amable Álvarez, I., Méndez Martínez, J., Delgado Pérez, L., Acebo Figueroa, F., de Armas Mestre, J., \& Rivero Llop, M. (2017). Contaminación ambiental por ruido. Revista Médica Electrónica [Online]. 39(3), pp. 640-649. Available at: http://scielo.sld.cu/scielo.php?script=sci arttext\&pid=S1684$18242017000300024 \&$ Ing=es\&tlng=.

Auger, N., Duplaix, M., Bilodeau-Bertrand, M., Lo, E., \& Smargiassi, A. (2018). Environmental noise pollution and risk of preeclampsia. Environmental Pollution, 239, pp. 599-606. https://doi.org/10.1016/j.envpol.2018.04.060.

Ballesteros, M. J., Fernández, M. D., Quintana, S., Ballesteros, J. A., \& González, I. (2010). Noise emission evolution on construction sites. Measurement for controlling and assessing its impact on the people and on the environment. Building and Environment, 45(3), pp. 711-717. https://doi.org/10.1016/i.buildenv.2009.08.011.

Ballesteros, S., Lorrio, S., Molina, I., \& Áriz, M. (2012). Contaminación acústica en el transporte sanitario urgente por carretera. Anales Del Sistema Sanitario de Navarra, 35(3), pp. 367-375. https://doi.org/10.4321/s1137-66272012000300002.

Berglund, B., Lindvall, T., Schwela, D. H., \& WHO. (1999). Guidelines for community noise. WHO. Available at: https://apps.who.int/iris/handle/10665/66217.

Bolaji, B. O., Olanipekun, M. U., Adekunle, A. A., \& Adeleke, A. E. (2018). An analysis of noise and its environmental burden on the example of Nigerian manufacturing companies. Journal of Cleaner Production, 172, pp. 1800-1806. https://doi.org/10.1016/i.jclepro.2017.12.007.

Cattaneo, M., Vecchio, R., López, M., Navilli, L., \& Scrocchi, F. (2013). Estudio de la contaminación sonora en la ciudad de Buenos Aires. Universidad de Palermo [Online]. 17(2), pp. 1-19. Available at: https://www.palermo.edu/ingenieria/PDFs/GIIS/Trabajo COINI Cattaneo1.pdf. 
Cohen, M. A., \& Castillo, O. S. (2017). Ruido en la ciudad. Contaminación auditiva y ciudad caminable. Estudios Demográficos y Urbanos [Online]. 32(1), pp. 65-96, 2017. Available at: http://www.scielo.org.mx/scielo.php?script=sci arttext\&pid=S018672102017000100065\&lng=es\&tlng=es.

Dabirian, S., Han, S. H., \& Lee, J. (2020). Stochastic-based noise exposure assessment in modular and offsite construction. Journal of Cleaner Production, 244, pp. 118758. https://doi.org/10.1016/i.jclepro.2019.118758.

D’Agosto, M. de A. (2019). Noise pollution, vibration, visual intrusion, and emission of solid and liquid waste. Transportation, Energy Use and Environmental Impacts, pp. 259-280. https://doi.org/10.1016/b978-0-12-813454-2.00007-6.

Degrandi, C. R., \& Nogueira, G. W. (2012). Exposición Ocupacional a la Contaminación Sonora en Anestesiología. Revista Brasileira de Anestesiología [Online]. 62(2), pp. 253-261. Available at: https://www.scielo.br/pdf/rba/v62n2/es v62n2a11.pdf.

Espinoza Vasquez, S., \& Jaramillo Arango, D. (2012). Diseño y construcción de una barrera acústica para reducir la contaminación auditiva generada por una perforadora de concreto en vías públicas. Facultad de Ingeniería, Ingeniería de Sonido, Universidad de San Buenaventura, Bogotá, Colombia. Available at: http://biblioteca.usbbog.edu.co:8080/Biblioteca/BDigital/72725.pdf.

Fernández, M. D., Quintana, S., Chavarría, N., \& Ballesteros, J. A. (2009). Noise exposure of workers of the construction sector. Applied Acoustics, 70(5), pp. 753-760. https://doi.org/10.1016/j.apacoust.2008.07.014.

Ganime, J. F., Almeida da Silva, L., Robazzi, M. do C. C., Valenzuela Sauzo, S., \& Faleiro, S. A. (2010). El ruido como riesgo laboral: una revisión de la literatura. Enfermería Global, (19), pp. 1-15. https://doi.org/10.4321/s1695-61412010000200020.

Garrido Galindo, A. P., Camargo Caicedo, Y., \& Vélez Pereira, A. M. (2015). Nivel de ruido en la unidad de cuidado intensivo adulto: Medición, estándares internacionales e implicancias sanitarias. Universidad y Salud, 17(2), pp. 163-169. https://doi.org/10.22267/rus.151702.1.

González Sánchez, Y., \& Fernández Díaz, Y. (2014). Efectos de la contaminación sónica sobre la salud de estudiantes y docentes, en centros escolares. Revista Cubana de Higiene y Epidemiologia [Online]. 52(3), pp. 402-410. Available at: http://www.redalyc.org/articulo.oa?id=223240764012.

Hammad, A. W. A., Akbarnezhad, A., \& Rey, D. (2016). A multi-objective mixed integer nonlinear programming model for construction site layout planning to minimise noise pollution and transport costs. Automation in Construction, 61, pp. 73-85. https://doi.org/10.1016/j.autcon.2015.10.010.

Martínez Suárez, P., \& Moreno Jiménez, A. (2005). El ruido ambiental urbano de Madrid: caracterización y evaluación cuantitativa de la población potencialmente afectable. Boletín de La Asociación de Geógrafos Españoles [Online]. (40), pp. 153-180. Available at: https://dialnet.unirioja.es/servlet/articulo?codigo=1308514.

MINEM. (2016). Reglamento de Seguridad y Salud Ocupacional en Minería. Decreto supremo N024-2016EM. El Peruano, pp. 595392-595447. Available at: http://extwprlegs1.fao.org/docs/pdf/per160277.pdf.

Mosquera, G. (2003). Base de Datos de Niveles de Ruido de Equipos que se usan en la Construcción, para Estudios de Impacto Ambiental. Thesis, Escuela de Ingeniería Acústica, Universidad Austral de Chile, Valdivia, Chile. Available at: http://cybertesis.uach.cl/tesis/uach/2003/bmfcim912b/doc/bmfcim912b.pdf.

Muralikrishna, I. V., Manickam, V., Muralikrishna, I. V., \& Manickam, V. (2017). Noise Pollution and Its Control. Environmental Management, pp. 399-429. https://doi.org/10.1016/B978-0-12-8119891.00015-4.

NTP. (2010). Acústica. Determinación de la exposición al ruido laboral. Método de ingeniería. Norma Técnica Peruana.

pp. 1-71. Available at: 
https://www.academia.edu/9753456/Determinación de la exposición a ruido laboral ISO 96 122010.

Pavón García, I. (2007). Ambientes laborales de ruido en el sector minero de la comunidad de Madrid: clasificación, predicción y soluciones. Universidad Politécnica de Madrid ETSII.

PCM. (2003). Reglamento de Estándares Nacionales de Calidad Ambiental para Ruido DECRETO SUPREMO No 085-2003-PCM. El Peruano, pp. 254090-254094. Available at: https://www.minam.gob.pe/calidadambiental/wpcontent/uploads/sites/22/2013/10/Reglamento-calidad-ambiental-para-ruido.pdf.

Rodrigo Fernando, L. C. (2013). Aplicación del método William Fine para la evaluación de riesgos laborales en motoniveladoras, cargadoras y bulldozers del Gobierno Autónomo Descentralizado de la Provincia de Chimborazo. Escuela Superior Politécnica de Chimborazo, Riobamba, Ecuador. Available at: http://dspace.espoch.edu.ec/bitstream/123456789/3148/1/85T00284.pdf.

Salas López, R., \& Barbosa Castillo, E. (2016). Evaluación del ruido ambiental en el Campus de la Universidad Nacional Toribio Rodríguez de Mendoza de Amazonas, Perú. Rev. Indes. [Online]. 2(1), pp. 88-96. Available at: http://revistas.untrm.edu.pe/index.php/INDES/article/view/69/183.

Saleh, S., Woskie, S., \& Bello, A. (2017). The Use of Noise Dampening Mats to Reduce Heavy-Equipment Noise Exposures in Construction. Safety and Health at Work, 8(2), pp. 226-230. https://doi.org/10.1016/j.shaw.2016.09.006.

WHO. (2018). Environmental noise guidelines for the European Region. World Health Organization, Europe. Available at: https://www.euro.who.int/ data/assets/pdf file/0008/383921/noiseguidelines-eng.pdf. 